that after removal of the cholesteatomatous masses or granulations which are frequently present in the antrum or mastoid cells, the otorrhea ceases and recovery takes place.

Sometimes, moreover, small circumscribed areas of inflammation exist in the mastoid process, which maintain the otorrhea and are inaccessible to ordinary modes of treatment. These are exposed by the operation and recovery is thus brought about more readily.

In connection with the two last-named indications, the operation acquires an exploratory significance, and under the modern favorable auspices of antiseptic surgical treatment, it may the more readily be undertaken on account of the danger which is always associated with a continuance of the disease.

255 South Seventeenth Street.

DISCUSSION ON THE PAPERS OF DRS. BRYAN AND TURNBULL.

Dr. C. H. BurnetT-I would like to ask Dr. Bryan whether anything had been used beside poultices and the Politzer bag? Dr. BRYAN - In the first case the man was in a very low con dition and could not give much information, but I understood that there had been only poulticing up to the time I saw him. In the other case there had been instillations of peroxid of hydrogen.

Dr. BuRnETT - In the histories of all the cases reported as acute otitis media followed by acute mastoiditis I can read between the lines that the mastoiditis is a secondary infection and in some cases I can see that the ear was over treated and secondary infection followed. I believe that in most instances the secondary inflammation was caused by the use of peroxid of hydrogen. Acute mastoiditis following otitis media has become much more frequent since the introduction of peroxid. You can imagine that erroneous ideas of treatment some people have, by one instance. I once found that a patient of mine, a physician, from whom I had removed the ossicles, had been for a year in the habit of cleansing his ears with his own saliva. I am sorry to say that nearly all the articles on this subject state nothing as to the treatment before the patient was seen by the aurist. I believe that cases of acute mastoiditis, complicating acute otitis media, are artificial results of improper irritative treatment and not the necossary result of the primary disease. They are instances of secondary infection due to the introduction and retention of morbific germs, by the irritative treatment of the primary inflammation and I know of no agent so efficient in forcing pyogenic germs into the inflamed ear as the expansive, energetic hydrogen binoxid.

Dr. J. A. StuckY - I have seen many cases of acute mastoid. itis caused by the use of peroxid of hydrogen in middle ear troubles, especially in acute otitis media. I consider it a dangerous remedy, and it is not right to use it in these chronic cases, unless there is a large opening.

Dr. MYLES-A coagulum of the mucoid substance and the peroxid is formed and is left in the recesses, from which it can not be washed out.

Dr. A. B. Randall-My experience has been in absolute conflict with much that has been stated. I must speak a word in defence of the Politzer bag, the syringe and peroxid of hydrogen. They are in constant use in my practice. While I have been reproached with the fact that I have a consid. erable group of mastoid cases to operate on, I will ask anyone to point out a case of mastoiditis as a primary cause. These cases are almost all taken from the practice of other men. I have never seen any evil results from my vicious habits. The proper employment of the Politzer inflation in the early stages of otitis media is of great value; its abuse has no place whatever. The use of peroxid of hydrogen is limited ordinarily to the acute cases. The perforation is commonly small, especially in children. When the hot douche has not given good results I have taken care that my peroxid should go into the very places where it is said to do so much harm. We have bad results when the peroxid does not penetrate. With the use of the intra-tympanic syringe, carrying the peroxid into the cavity so that it bubbles out at the nose, I have seen these cases go on to rapid recovery. While I do not advocate this as a routine treatment, I have no fear as to the peroxid, and believe that it should be more widely used, especially in Europe where it is hardly known. Cases of the type mentioned by Dr. Bryan sometimes occur with peculiar frequency in the experience of some men. They are by no means as rare as is sometimes supposed. I have had perhaps twenty or twenty-five of them, and my assistant, Dr. Hammond, has had eight or ten. The cases to which we have reference today are acute.

Dr. Cline-I had a case in which I removed a large polyp from the external auditory canal. Later, when the patient was returning to a neighboring town, he had a chill on the road. He came under the care of a very good aurist, who for a week worked hard to save his life. There was inflammation of the mastoid cells. The doctor, I understood, had washed out the cavity with peroxid and had put something into the ear to stop the discharge suddenly. I believe that we should discriminate in our cases. If we have a large perforation we can use peroxid with more safety than with a small opening, but it must be used carefully.

The Chairman - I would ask Dr. Burnett if he objects to the use of peroxid in chronic cases to the same degree.

Dr. BuRnETT-I do not use peroxid at all, either in acute or chronic suppuration of the ear on account of its expansive force and the consequent risk of forcing pus into the middle ear cavities. It is perhaps less harmful in chronic than acute cases. We must regard the antrum as part of the middle ear, not the mastoid. Many acute cases will get well without mastoid disease. A natural siphonic action takes place between it and the antrum. When I was a medical student we never heard of mastoid disease occurring as a sequel of otitis media. Why should one man now see 100,200 or 300 cases in a comparatively short time if it is a necessary result of ear disease? The so-called acute mastoid disease as a sequel is largely an artificial result.

H. D. HATCH, D.D.S. - I once had a lesson as to the explosive force of peroxid of hydrogen. In working on a tooth my instrument slipped up under the tissues, almost to the eye. The wound healed and was apparently all right, but later an abscess formed, well up toward the eye. There was no swelling around the eye, except this small point, and there was no wound in the mouth. I slipped a bistoury in to the abscess and injected peroxid of hydrogen. Inside of twelve hours after my injection, which caused intense pain, the face was swollen and the eye black. I have ceased to inject peroxid into any cavity into which there is a small opening, except in some cases where I inject a little at a time and tease out the pus. I have had other cases where the mechanical force of the explosion gave great pain. Members of the dental profession use peroxid of hydrogen largely. I came to to the conclusion last winter that it was unwise to inject it into a bone cavity.

Dr. BRYAN - I have seen serious results from the use of the Politzer bag and from the use of peroxid of hydrygen, but they were at the hands of operators who did not know how to use them. My object was not so much to advise the members of this Association against the use of these things as to urge their cautious use. I have thought it best to present these cases so that they might be an object lesson to those who are not so careful.

\section{PHENOMENA OBSERVED IN TWELVE CASES AT VARIOUS STAGES OF THE OPERATION FOR SECTION OF THE INCUDO-STAPEDIAL ARTICULATION AND MOBILIZATION OF} THE STAPES.

Read in the Section on Laryngology and Otology, at the Forty-eighth Annual Meeting of the American Meaical Association held at Philadelphia, P'a., June 1-4, 1897. BY E. B. GLEASON, M.D.

Clinical Professor of Otology in the Medico-Chirurgical College; Surgeon-in-Charge of the Nose, Throat and Ear Department of the Northern Dispensary, Philadelphia.

PHILADELPHIA, PA.

The ear in which the patient's hearing was the more defective was invariably selected for operation. The night before the operation the auditory canal was thoroughly cleansed, syringed gently with a solution of bichlorid, 1 to 1000 , and occluded with a piece of iodoform gauze, which was allowed to remain in position over night. The drumhead was soaked for one hour before the operation in a sterilized 4 per cent. 
solution of cocain, by means of a pledget of sterilized absorbent cotton, which was saturated in the solution and inserted into the canal in contact with the drumhead. After the primary incision through the membrana, the edges of the wound and the intratympanic membrane was touched from time to time with a sterilized 10 per cent. solution of cocain. All maneuvers subsequent to the primary incision were thus rendered practically painless.

The steps of the operation performed for the relief of tinnitus and improvement of the hearing, in twelve cases of advanced sclerotic catarrh of the middle ear that had not improved as the result of non-operative measures, were as follows: An incision through the clear part of the membrana, as close to the periphery as possible, was made from a point below the level of the malleus tip to the short process, and the flap thus produced was turned forward over the malleus handle. An attempt was made to mobilize the chain of ossicles by traction in various directions upon the lower portion of the long process of the incus. In the last six of the twelve cases operated upon, the tendon of the stapedius muscle was then severed and the attempt renewed to mobilize the chain of ossicles by traction upon the long process of the incus. The incudo-stapedial articulation was next severed and the stapes mobilized if possible by lateral pressure and lever-like movements of a cotton-tipped probe in contact with the head of the stapes. In the last of the series of cases, sufficient force was exerted to fracture the crura of the stapes; but in this case not the slightest improvement of the acuteness of hearing followed the operation. There were however no disagreeable after-effects.

In all but one case the operation was comparatively bloodless. In this instance, however, such a persistent hemorrhage followed the primary incision through the drumhead that the operation was abandoned. Two weeks afterward an opening was made through the membrana over the incudo-stapedial articulation, by means of a cotton-tipped probe dipped in fuming sulphuric acid, the articulation severed, and the stapes mobilized. The slight improvement of the hearing that resulted from this procedure was lost during the course of an otorrhea that persisted for three or four weeks after the operation, but the patient was cured of an annoying tinnitus. Suppuration did not occur in any of the other cases.

Each of the twelve cases operated upon in the manner described above had their hearing carefully tested both with voice and watch at each step of the operation.

In no instance did the slightest improvement of the hearing follow the incision of the drumhead and the turning forward of the flap. Although the evidence of twelve cases is by no means conclusive, yet the writer can not help suspecting that the idea that improvement of hearing in catarrhal cases with patulous Eustachian tubes would follow the mere excision of a portion of the drumbead is a myth, and that the improvement of hearing that was reported to have followed this procedure was due in all probability to incidental mobilization of the chain of ossicles. This suspicion becomes more plausible when it is called to mind that Sir Astley Cooper did his operations with an ordinary trocar, and that up to the time of Wilde, the favorite instrument for performing the operation was that of Fabrizzi, consisting of a small trephine with a cork screw stilet, by means of which not only a section of the membrana, but also a portion of the malleus handle was usually removed. So inadequate was this instrument for the safe perforation of the drumbead that two deaths following the operation were reported, in London, by Dr. Butcher in 1846, and in these two cases, at least, it is probable that something more was accomplished by the operation than the mere removal of a disc of the drumbead and a most thorough mobilization of the ossicular chain.

It also seems probable that in many instances the improvement of hearing and relief of tinnitus reported as following the modern operation for the removal of the drumhead, malleus and incus, were the result of the incidental mobilization of the stapes. The only advantage in catarrhal cases of such a procedure over the operation described above, consists in the accessibility of the stapes for purposes of mobilization, which is probably more than counterbalanced by the constant exposure of the intra-tympanic mucous membrane to the danger of infection by pyogenic bacteria.

It was found impossible to improve hearing in any of the twelve cases by manipulation of the long process of the incus in the hope of mobilizing the incus. Section of the stapedius muscle was accomplished, in two instances, with an audible snap and an immediate improvement of the hearing In both cases, however, further improvement followed the subsequent incision of the incudo-stapedial articulation and mobilization of the stapes.

The amount of force used where no improvement of hearing resulted from the operation, was in all instances sufficient to endanger fracturing the crura of the stapes, which actually occurred in the last case operated upon. In this and others where no improvement of the hearing followed manipulation of the stapes, it is probable that ankylosis of the foot plate of the stapes to the oval window existed to such a degree as to render it absolutely impossible to break up the adhesions without fracturing the crura in the attempt. Politzer has proven by his experiments upon the cadaver that when bony ankylosis exists between the foot plate of the stapes and the oval window the crura are invariably broken in an endeavor to remove the stapes by traction.

The duration of the vertigo which invariably ensues in the cases where improved hearing followed manipulation of the stapes, varied in duration from a few moments to three days. One case was obliged to lie down three times during the manipulation of the stapes because of dizziness and faintness, which, however, lasted only a few moments. In another instance the patient staggered almost like a drunken man as he was led away from my office at the conclusion of the operation. His dizziness lasted only twenty-four hours.

In no instance did any permanent injury to the auditory apparatus follow the operation. In all cases where tinnitus existed, it disappeared after the operation. There was, however, only in five a noticeable and practical improvement of the hearing. In one, improved hearing for the voice did not entirely disappear until three years after the operation. In all cases improved hearing for the watch invariably disappeared within a few months, probably as the result of the reformation of adhesions. In one or two hearing notably improved both for watch and spoken words for a few weeks subsequent to the operation. It seems more than possible that the hearing might have been again improved in some at least of the 
cases by incising the drumhead and remobilizing the stapes.

41 South 19th St.

\section{DISCUBSION.}

Dr. BuRnETT. I have seen several cases of perfectly mobile stapes with total deafness, and total ankylosis of the stapes with very good hearing. I think that so far the operation can claim to relieve chiefly in cases of aural vertigo. These cases can of ten be treated successfully by intra-tympanic operation, either by Dr. Gleason's method or, as I prefer, by the removal of the incus alone.

Dr. RANDALL-We may properly hope for this operation a place equal in importance to that held by the operation for cataract in the surgery of the eye. There, in a large number of cases, lies the impediment to the betterment of the organ of hearing. More attention should be paid to the proper testing of hearing in these cases. Disappointmente will greet us for a long time, as they have done in the past, as in the case of the operation for the removal of the drumhead, which a few years ago was exploited as the new treatment for all ear diseases. Even with great care and proper selection of cases, results will often be very disappointing.

Dr. TuRnBulL-I feel as if $I$ had been an advocate of the removal of the malleus. One of my cases was operated on seven years ago and her hearing is still good. She was very deaf indeed. The benefit may have come from the mobil. ization which attended this operation. In another case I operated on a lady who had suffered greatly with vertigo. The vertigo was entirely relieved and she has fairly good hearing.

Dr. BERNSTEIN-I have removed the incus in three cases, but I shall never do the operation again. In one case, for about three weeks after operation the patient's hearing was decidedly improved, but after that she had to use a trumpet. This has been my experience in the other cases also.

Dr. MyLES-I have rather opposed the removal of the ossicles. When cures from this operation are reported, it is not always stated how long the hearing remains improved. I know one man whom a distinguished aurist persuaded to have his ossicles removed, and for awhile his hearing was improved; but now he can hear nothing. Two young men came to me two years ago, whose hearing had been much impaired for about two months. I found large exostoses and complete nasal stenosis, and one of them had considerable adenoid tissue and subacute otitis media. I advised rest. One of the young men told me that he had come from the office of a distinguished aurist who had been almost violent in his efforts to induce him to have the ossicles removed. I advised them both to wait and try the rest treatment first, which they did. They both hear well today. If the ossicles had been removed, they would have heard better for a time, and would probably have been reported as remarkable cures.

Dr. GLEason-Until a couple of weeks ago, I supposed the result I obtained in a case operated on two years ago was per. manet, but the patient told me that all the improvement had disappeared. I would advocate this operation only after everything else has been tried.

\section{OBSERVATIONS ON SOME PATHOLOGIC CONDITIONS OF THE NASO-PHARYNX.}

Presented to the Section on Laryngology and Otology at the Fortyeighth Aunual Meeting of the American Medical Association, at Philadelphia, Pa., June 1-4, 1897.

BY EMMA E. MUSSON, M.D.

PHILADELPHIA, PA.

Believing that the presentation of cases having either special pathologic features or dependent on unusual causes, or amenable only to special lines of treatment, would be of interest. I have selected from my case book several groups of cases which have come under my observation during the past two years.

The first group comprises a number of cases of hypertrophied lymphoid tissue in the vault of the pharynx, all of which required for their relief operative interference. The points of interest in these cases, relate to the age and the character of the symptoms. Out of the last 230 patients in my case book, 34 had hypertrophy of the third or Luschka's tonsil.
Of this number eleven were from 15 to 20 years of age; eleven from 20 to 25 ; eleven from 25 to 35 , and one 40 years of age. The age of the majority of these patients attracted my attention on account of the prevalent belief that these lymphoid vegetations are peculiar to children and disappear at the period of puberty.

Of these thirty-four cases, nine were complicated with profuse post-nasal discharges. In five instances the growth interfered with singing upper notes; in one, with elocutionary work and in one with ordinary school teaching.

Two cases were complicated with hay fever; two with mouth breathing; two were subjects to attacks of acute pharyngitis and tonsillitis, and two were subject to frequent colds.

Twelve cases were complicated with disturbance of the ear. Of these, three had marked deafness; three were moderately deaf; one had purulent otitis media; two acute Eustachian catarrh and three tinnitus and fulness.

The majority of these cases had post-nasal catarrh. catarrh. The hypertrophied lymphoid tissue in at least 95 per cent. of the cases was entirely confined to the vault of the naso-pharynx. The growth varied in appearance and extent, from apparently smooth masses, one quarter of an inch in thickness to vegetations as large as those found in well marked cases in children. A number of these vegetations were connected with the Eustachian tube by synechiæ. Moreover in those cases complicated by diseases of the middle ear, the vegetations were fibrous in character while in those complicated by a profuse muco-purulent serretion, they were soft and friable.

Surgical procedures for complete removal of the growths, were followed by relief if not complete disappearance of all the symptoms except the deafness. There was a disappearance of the post-nasal discharges and of the seeming purulent rhinitis, and the patient was no longer subject to repeated colds and sore throat. The voice became more resonant in speaking and two or three notes were added to the upper register of the voice in singing.

Surgical interference which gives such brilliant results in early childhood, for the relief and cure of deafness, was in my cases followed only by slight if any benefit; not until after a long course of patient treatment of the middle ear disease was any improvement noticed. In those cases however in which the middle ear was but slightly involved and the symptoms present only at intervals a prompt cure was effected upon removal of the growths. Of the cases complicated with naso.pharyngeal secretions, four were of exceeding interest. In two of them the discharge from the nose and naso-pharynx was purulent, profuse and constant. In the two other cases the secretion was excessively profuse, but entirely mucoid in character. Three had a family history of gout and rheumatism, and had not yielded to prolonged treatment for nasal catarrh. In two the amount of lymphoid tissue was so small that I hesitated long before removing it; there was an entire absence of the gross appearance of adenoid growths, the vault of the pharynx showing only a thin sheet of lymphoid tissue. After the lapse of a year, during which there was no recurrence of the discharges, I felt that I had been justified in my operative procedures.

The methods resorted to for the removal of these growths were the forceps followed by curetting in the 